\title{
Efficient Applications of Risk Analysis in the Chemical Industry and Emergency Response
}

\author{
Roberto Bubbico \\ Dipartimento di Ingegneria Chimica - Università di Roma "La Sapienza” \\ Via Eudossiana 18, 00184, Roma (Italy)
}

\begin{abstract}
Despite it is now used in many technical and industrial areas, Risk Analysis is sometimes still considered by many plant managers as a methodology too complex and too timeconsuming to be of practical use. Conversely, it will be shown that Risk Analysis can often provide very useful information on the safety of selected industrial activities. Also, the introduction of proper simplifying assumptions can even wider the range of application with great benefit of all stakeholders.
\end{abstract}

Keywords: Risk Analysis, Safety, Chemical industry, Hazardous materials, Emergency response

\section{Introduction}

The introduction of the first techniques for the calculation of the risk posed by a given technical activity, dates back to the end of the 1970s in the area of the nuclear and aeronautics industries. Some of them are nowadays quite well known among practitioners, such as the Probabilistic Risk Assessment (PRA) ${ }^{1,2}$, the Preliminary Hazard Analysis (PHA), and so $\mathrm{on}^{3,4}$. However, in the recent years, these techniques, or some modifications of their original versions, have been adopted to a large extent, also in many new areas, such as finance, medicine, biology, etc., different from the native ones ${ }^{5-7}$.

This is because of the high flexibility of the methods and to the capability of a very detailed and powerful analysis of the system under study, whether an industrial installation, an informatics network, a financial system and so on.

Among the many methodologies available, the quantitative approaches are able to provide more detailed and useful in- 
formation but, on the other hand, they are usually much more time-consuming and demanding in terms of computational effort. Furthermore, due to the uncertainties in the input data or to the lack of adequate information, in some cases the accuracy and reliability of the results are a matter of discussion.

Despite these difficulties, Risk Analysis has gained a good reputation among practitioners, especially in its quantitative version (Quantitative Risk Analysis, QRA) $)^{8,9}$. With specific reference to the area of the chemical and process industries, the main problems involved in its use will be here critically analysed and a number of useful examples of application presented.

\section{Risk Analysis}

Risk Analysis is a powerful and comprehensive methodology that can be adopted to assess of the level of the risk associated with a given activity (industrial, economical, etc. $)^{5,6}$. It is a stepwise procedure and more than one single component technique are available for performing each of the tasks within the whole procedure.

The need for a stepwise procedure is given by the many aspects which must be considered to estimate the value of the risk. These aspects depend on the area of application, the risk possibly being linked to a financial loss/income, to the safety of an industrial activity and so on. In the specific case of the chemical industry, different variations can be found in the definition of risk in the literature (see for example the paper by Kaplan and Garrick $^{9}$, for a more thorough analysis), but it is generally agreed that risk is a function of a combination of the impact of a number of selected hazardous events, and of their probability/frequency of occurrence. Of course, based on this definition, the value of this function strongly depends on the identified harmful events (accident scenarios). As a consequence, in order to estimate the risk, the following four traditional main steps composing the whole analysis are required:

- Hazard identification

- Consequence calculation

- Frequency estimation

- Risk evaluation

As mentioned above, in order to accomplish each of these steps, different techniques can be adopted, thus providing the methodology with a high level of flexibility. In fact, on one hand, the methodology can be applied to any stage of the lifetime of an industrial installation: from the initial preliminary design stage, to engineering phase, up to the actual operation of the plan, possibly taking into account all structural and procedural changes (management of changes). On the other hand, the methodology can be applied to a variable level of detail for the same stage of the plant: all the specific pieces of equipment can be studied, along with the associated accidents and their frequency of occurrence and magnitude of consequence; or larger subsystems of the plant can be, at least preliminarily, considered as a black box, to allow a more general and quicker analysis to be carried out. Of course, once the critical subsystems have been identified, they can be analysed to a higher level of detail.

Another very important aspect is that the methodology can be either qualitative or 
quantitative. From this point of view, it worth noting that different conclusions can be drawn by these two approaches. A typical example: it is generally assumed that an accident deriving from one single initiating event, is more critical than one occurring from the combination of two or more initiating events. This sounds reasonable. However, when quantitatively analysed, the frequency of occurrence of the first accident can result even much smaller than that of the second one, thus revealing a misleading conclusion of the qualitative approach. Therefore, when planning a risk analysis study, it is important to clearly state since the beginning the scope and aim of the analysis.

Besides this aspect, it must be said that some of the techniques and models used for performing these tasks can be rather complex and often require skilled and experienced personnel. And even in the case of relatively simple models, a minimum level of uncertainty and approximation is implicit.

As examples of such complex models, in the area of consequence calculation, the mathematical models adopted for the assessment of the fraction of aerosol generated after a liquid release, or those used for the calculation of the dispersion of a heavy gas, can be mentioned. In the case of the dispersion of a heavy gas in a complex environment, such as a urban area or a congested industrial site, the application of these models becomes even more difficult and usually require a trained analyst, familiar with the fluid-dynamics and thermodynamics.

Toxicological information are available only for a limited number of substances, and therefore, even when dispersion can be assessed rather accurately, effect models cannot be used without a high level of uncertainty.

Other difficulties arise when there is a lack of historical data required as input parameters for some models. For example, the values of the frequency of occurrence of some events (release of a material from a containment system, rupture or failure of a piece of equipment, the ignition of a flammable mixture, and so on) are hardly found in the literature, or, when available, they are referred to systems/conditions other than those under investigation. In these cases the needed parameters have to be estimated by means of specific techniques (Fault Tree Analysis, Event Tree Analysis, etc.), which represent another important source of uncertainty. In fact, besides the quality of the data used to quantitatively solve these models, even the definition of the model itself is prone to uncertainties and errors.

Based on the above considerations, it appears that the inherent structure of Risk Analysis, on one hand gives rise to a very flexible and powerful methodology for the assessment of the safety of an industrial activity, but, on the other hand, is a source of uncertainty and errors. Also, the higher the accuracy of the results required, the longer the time and the calculation burden for performing the analysis. Just to represent more clearly the uncertainties we are dealing with in this area, it can be useful to mention a famous study carried out in the past at a European lev$\mathrm{el}^{10,11}$ : a number of Risk Analysis teams were provided with the same system to analyse, with specific indications on the techniques to use, and so on. Yet, the 
possible assumption of hypotheses, the selection of the incidents to study and of the input data (frequencies, and so on) were on their own judgment. At the end of the study, the results obtained by the different teams ranged over several order of magnitudes, and the influence of the many uncertainties was not enough to explain this variability. Only when much more restricting instructions were given to the teams, a much more acceptable variability was obtained.

Based on these considerations, a strong debate sometimes arises among practitioners, especially process engineers and plant personnel, about the actual reliability and effectiveness of the methodology. Different arguments are also debated in the literature ${ }^{12,13}$.

Despite these difficulties and sources of uncertainties, Risk Analysis still represents a useful and sometimes unique methodology for the assessment of the safety of a given industrial activity, especially when a comparative use is to be carried out: e.g. when different layouts of the plants or alternative production processes have to be evaluated. This is now generally recognized and in fact the use of this technique is nowadays also recommended by different regulations in force in Europe $^{14}$ as well as in other Countries all over the world.

\section{Examples of application}

In the following paragraphs, a number of examples of application of the methodology to various industrial activities will be reported. However, besides the "conventional” use of Risk Analysis, it must be noted that its component techniques are widely used in a number of other applica- tions: consequence evaluation ${ }^{15-17}$, decision making ${ }^{18-20}$, process $\operatorname{design}^{21}$ and others.

\subsection{Transportation Risk Analysis}

Any process industry managing hazardous substances as raw materials or final products to be sent to the market, requires the transportation of these materials to/from the production site. This specific task has to be considered as an integral part of the general production activity. As a consequence, in the framework of the assessment of the risk connected with the whole production activity, the analysis of the risk associated with the transportation phase has to be taken into consideration along with that connected with the other production units. This has been recognized recently, and a specific version of the original Quantitative Risk Analysis technique has been set up, usually referred to as Transportation Risk Analysis (TRA) ${ }^{22}$. This is an extension of the traditional quantitative risk analysis technique, with the distinctiveness that the risk source is not in a stationary location, but is moving along a given route in a continuously changing environment. Therefore, in order to apply the technique to transportation activities, the knowledge of a large amount of information is required, partly strictly connected with the territory characteristics, and partly relative to other parameters. Just to mention a few examples: the local distribution of population along the route, the site-specific accident rates, the local weather conditions, the accidents evolution in relation with the local specific characteristics, and so on. The need of this considerable amount of information often prevent the application 
of this very useful methodology to practical cases, with heavy detriment of the safety for the involved working personnel, for the exposed population or for the environment.

In order to overcome these complexities, different approaches have been proposed in the literature, and a number of methods of implementation of the technique have been devised (see [15] for some references).

In most of the cases, the proposed variations are simplified versions of the more rigorous approach ${ }^{23,24}$. For example, a first solution can be to distinguish the parameters which are really routedependent from those which are not ${ }^{23}$. In the first group can be considered the release scenarios, the probability of a release scenario after a given accident and the probability of an outcome case following a given release scenario.

Other parameters, such as the accident rate, the population distribution and the weather conditions, have to be considered as route-dependent factors. However, some simplifying assumptions are possible also in this case, at least as a first approximation: the accident rate can be considered as a function of the type of road (highway, state-road, etc.); typical values of population densities can be associated to a reduced number of different types of built-up areas ranging from urban to rural, and so on. All these assumptions allow to reduce the amount of information to be collected.

Of course, each simplification will involve advantages and disadvantages. In summary, the pros consist in the availability of a less demanding procedure, requiring a reduced amount of input data, and in a more "user-friendly" approach, allowing the technique to be applied even by less experienced practitioners. On the other hand, the use of too many or too "heavy" simplifications can lead to a less than acceptable accuracy of the results; this can finally result in the adoption of erroneously biased decisions during risk management.

Even if the accuracy of the results cannot be expected to be very high, in some cases, such as a preliminary analysis, it can provide very useful information and allow an efficient use of time. In Figure 1 a comparison between a simplified and a rigorous approach is shown.

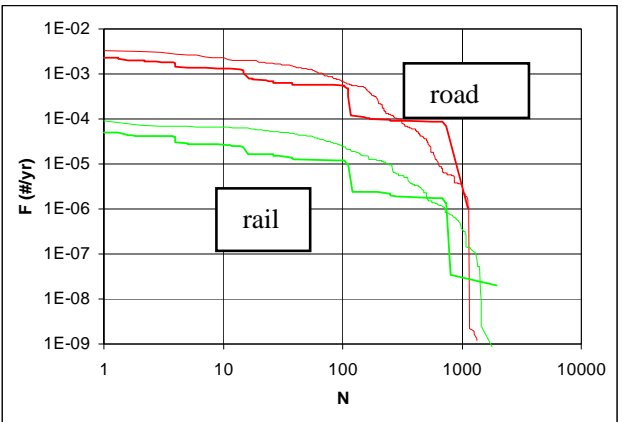

Fig. 1. Comparison of F-N curves obtained by a simplified and a rigorous approach [10]

In fact, a similar simplified approach has been proposed in the literature ${ }^{25}$ to identify critical areas along a specific route and thus provide effective strategies, such as a different distribution of the dangerous goods traffic, alternative routes and/or limitation to the transportation itself. Also, the adoption of techniques based on the graph theory allowed to improve the planning of the emergency phase.

A more efficient way to manage the TRA calculations involves the use of Geographic Information Systems (GIS). By 
this methodology, a tool capable of performing in a simple and relatively quick way all the steps of a TRA can be devised.

The consequences of an accident are influenced by many parameters, and some of them depend on the specific location (e.g. accident rate, on-route and off-route population, weather conditions and so on). GIS databases are able to provide some of these data already directly linked to the location along the route network. Different commercial GIS databases are already available for almost any Country in the world, providing at least some basic information (road and rail network, land use, and so on).

Nonetheless, the values of other important parameters (accident rates, population and meteorology) have to be collected from different sources and then manually introduced in the GIS system. Depending on the available data and their initial format, these phases (data acquisition and manipulation) may be quite long (e.g. see [26, 27]). Besides the GIS system, a database containing information on the probabilities of occurrence of all the possible accident outcomes, and on the magnitude of the consequences of such dangerous events under the selected reference weather conditions, has to be prepared. Of course, these data are available only after the chemical products of interest and the weather conditions to be associated with the considered accident have been defined. The larger the number of chemicals and/or the number of weather conditions adopted, the "heavier" the database and the more accurate the calculation results. By such a GIS system, the calculation procedure is very simple: once the product and the schedule of the transportation (number of trips per season) have been defined, and once the route of interest has been selected, all the related data are automatically uploaded by the software and used for the calculations. In Figure 2, the selection of a specific route between given origin and destination is shown.

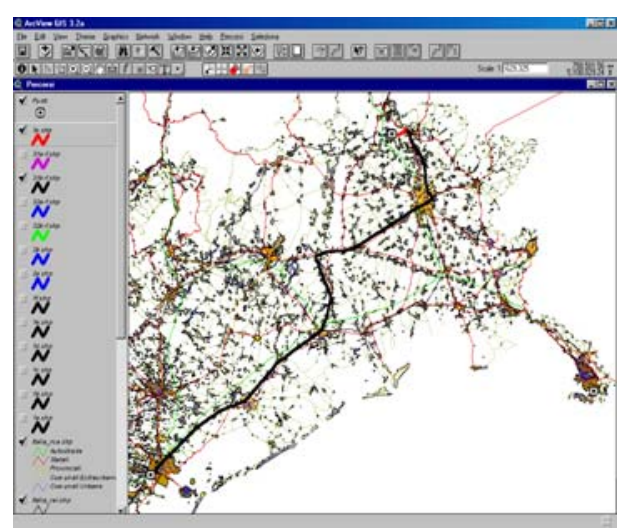

Fig. 2: Selection of the route of interest on the GIS map [26]

The local information about many different parameters (population, road characteristics, etc.) can be automatically displayed on the same map by simply clicking on the proper GIS tool buttons (Figure 3).

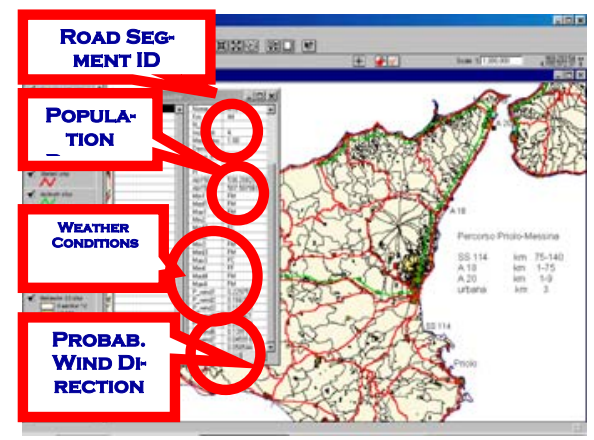

Fig. 3: Site-specific information on the GIS map [26] 
Both the individual risk as a function of the distance from the route, for each route segment, and the societal risk, in terms of the cumulative F-N curve of the route, can be finally displayed (see Figures 4 and 5).

Sometimes, the procedure can be also speeded up by using one of the GIS selection tools, such as the fastest or shortest route selection option.

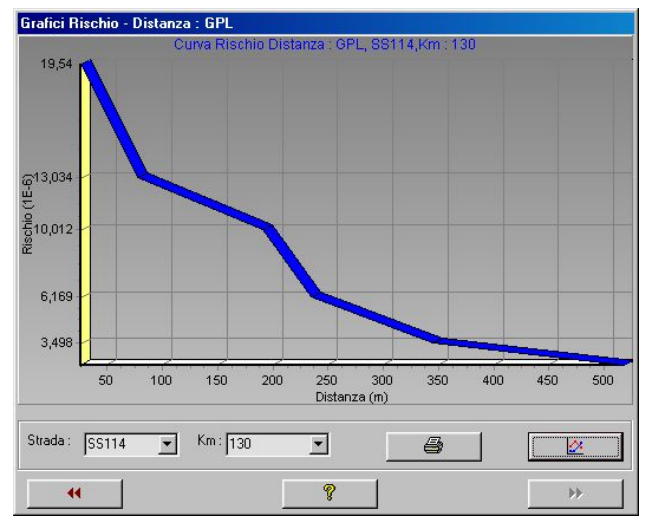

Fig. 4: Individual risk as a function of the distance from the road.

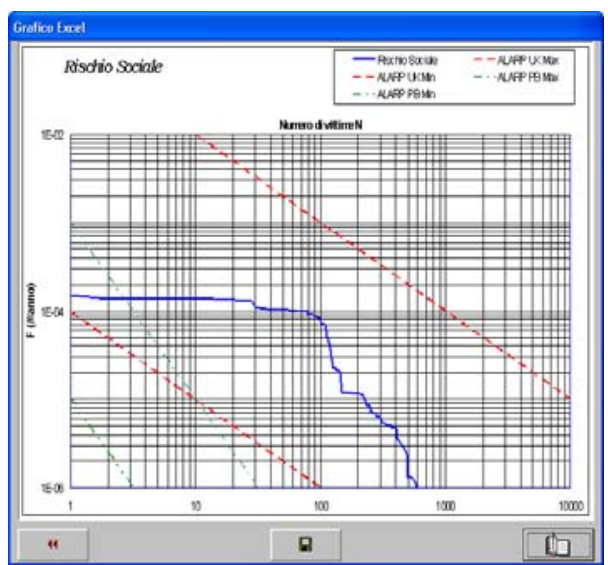

Fig. 5: Societal risk: F-N curve for the whole route selected.

If different routes can be chosen for the same transportation activity, the construction of the risk curves for each of them would allow to identify the one which is characterized by the lower level of risk. At the same time, the knowledge of the individual risk profiles along the selected route make it possible to locate the most hazardous spots: this would allow, for example, to slightly modify the route, achieving a lower risk value with minor changes and, correspondingly, with negligible cost increases.

It is thus apparent that such a tool is able to permit a fast and relatively accurate investigation of the alternative routes or transportation modalities for a given activity, and to provide a comprehensive risk management tool.

Different examples of application can be found in the literature. Here, reference will be made to a previous study ${ }^{28}$ where the overall risk associated with the transportation activities of hazardous materials in a whole region of Italy has been reduced by simply modifying the distribution of the transported chemicals in terms of transport modalities and schedule.

The data concerning the transportation of dangerous goods in Sicily (Italy) were obtained from different sources (Civil Defence Office of the Prefettura of Messina, Federchimica, Italian railways company, harbour offices, etc.). Also, the routes travelled by the products were identified with a reasonable degree of accuracy, thus allowing to get the specific accident frequencies and other characteristics of the travelled routes. The analysis of the data allowed to identify 51 road transportation cases and 4 rail ones, and the use of a specifically devised GIS tool led to the construction of the following overall F-N curve (Figure 6): 


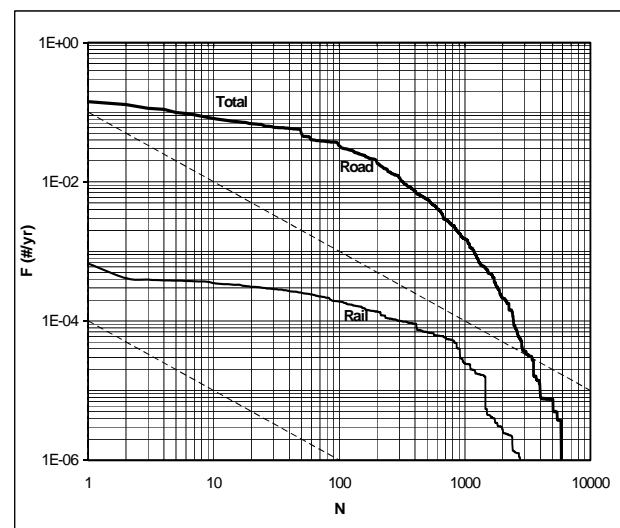

Fig. 6: Societal risk for the initial transportation activity.

By re-arranging and optimizing the distribution of the transport among road, rail and intermodal and, consequently, modifying the amounts of materials transported and the routes travelled, a new risk curve is obtained. The advantages of the new organization of the transport, is demonstrated by the risk reduction shown in Figure 7, where a comparison between the two curves (before and after redistribution) is reported.

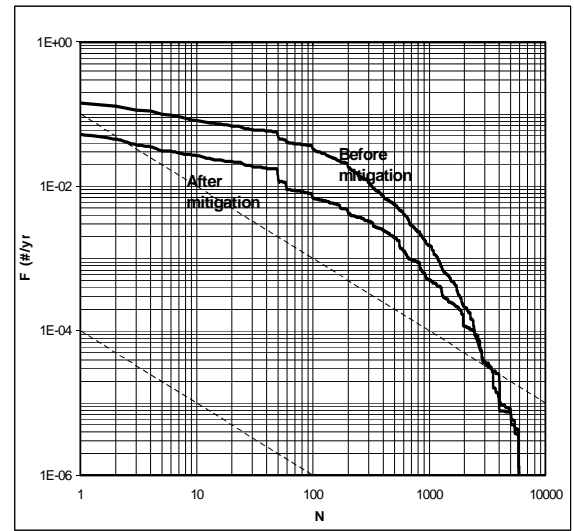

Fig. 7: Societal risk for the optimized transportation activity.

\subsection{Emergency support tool}

A GIS application quite similar to that set up for the analysis of the risk associated with the transportation of hazardous materials, can represent a useful support tool in the case of an emergency. In fact, in the case of an accident, if its location is identified on the relevant GIS map, the impact areas of all the possible outcome cases associated to the corresponding accidental scenario (dependent on the substance, the type of release, the environmental conditions, etc.) can be easily displayed on the map itself. If specific information, such as the actual wind direction at the time of the accident, are available, a rather accurate prediction of the territory at risk (of fire in the case of a flammable release, of toxic exposure in the case of a toxic cloud, etc.) can be identified and very useful information can correspondingly be obtained: e.g. the urban areas to be evacuated by the population, the roads and/or railways to be prohibited or, conversely, those to be used by the emergency teams for reaching the location of the accident; similarly, if the location of centres of interest (fire brigade and police stations, civil protection offices, hospitals, etc.) are reported on the map, it might be possible to know what centres need to be activated following the emergency. In Figure 7 the possible impact areas following the release of ammonia from a road tank are represented.

The examples reported here are only a few of the many possibilities offered by such a kind of tool. 


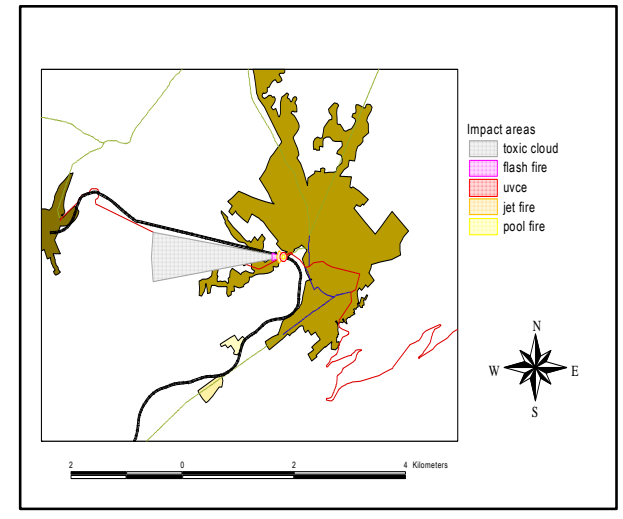

Fig. 8: Impact areas following a release of ammonia from a road tank.

\section{Conclusions}

A quick presentation of some of the most common applications of the Risk Analysis technique in the industrial sector and in the civil protection and emergency planning area has been reported in the present paper.

It should be apparent that, despite the complexity of the procedure and the considerable effort in terms of calculation and data collection, Risk Analysis still represents in many cases the only method able to provide useful information on the safety and quality of a selected industrial activity. It represents a quantitative approach and, despite the absolute values obtained cannot be considered as "the" value of the investigated risk, especially when applied in a comparative analysis, the results provided can be trusted with a sufficient level of confidence.

Furthermore, when a number of appropriate simplifying assumptions are introduced, the application can be definitely easier and thus the range of applications and of possible users becomes quite larger with great benefit for all stakeholders: the responsible of the activity under investigation and the exposed population.

\section{References}

1. NUREG, U.S. Nuclear Regulatory Commission, "PRA procedures guide: a guide to the performance of probabilistic risk assessment for nuclear power plants”, NUREG CR-2003, Washington D.C., 1984.

2. NUREG, U.S. Nuclear Regulatory Commission, "Probabilistic safety analysis procedures guide”, NUREG CR-2815, Washington D.C., 1985.

3. N. Hyatt, "Guidelines for Process Hazards Analysis, Hazards Identification \& Risk Analysis”, Dyadem Press, Richmond Hill, Ontario, Canada, 2003.

4. CCPS, Center for Chemical Process Safety, "Guidelines for hazard evaluation procedures”, 2nd edition, AIChE, New York, 1992.

5. G. Koller, "Risk Assessment and decision making in business and industry- A practical guide”, Chapman and Hall/ CRC, Boca Raton, FL, 2005.

6. T. Aven, O. Renn, "The role of Quantitative Risk Assessment for characterizing risk and uncertainty and delineating appropriate risk management options, with special emphasis on terrorism risk", Risk Analysis, Vol 29/4, pp. 587-600, 2009.

7. H. Li, G.E. Apostolakis, J. Gifun, W. Van Schalkwyk, S. Leite, D. Barber, "Ranking the risks from multiple hazards in a small community”, Risk Analysis, Vol 29/3, pp. 438-456, 2009.

8. CCPS, Center for Chemical Process Safety, "Guidelines for Chemical Process Quantitative Risk Analysis”, 2nd edition, AIChE, New York, 2000.

9. S. Kaplan, B.G. Garrick, "On the quantitative definition of risk”, ”, Risk Analysis, Vol 1/1, pp. 11-27, 1981

10. Amendola, "Uncertainties in systems reliability modeling: insight gained through European benchmark exercise”, Nuclear Engineering Design, 93, 215-225. Amsterdam, The Netherlands, Elsevier Science, 1986 
11. Amendola, S. Contini, I. Ziomas, "Uncertainties in chemical risk assessment: results of a European benchmark exercise", J. of Hazardous Materials, 29, 347-363, 1992

12. Z. Ciechanowicz, "Risk analysis: requirements, conflicts and problems", Computers and security, 16/3, 223-232, 1997

13. M. Elisabeth Paté-Cornell, "Uncertainties in risk analysis: six levels of treatment", Reliability engineering and systems safety, 54, 95-111, 1996

14. European Commission Council Directive, 96/82/EC, On the control of major accident hazards involving dangerous substances, 9 December 1996

15. J. Tixier, G. Dusserre, S. Rault-Doumax, J. Ollivier, C. Bourely, "OSIRIS: software for the consequence evaluation of transportation of dangerous goods accidents", Environmental Modeling \& Software, Vol. 17/7, 627-637, 2002

16. R. Bubbico, M. Marchini, "Assessment of an explosive LPG release accident: A case study.” J. of Hazardous Materials, 155, pp 558-565, 2008

17. P.A. Davies, F.P. Lees, "The assessment of major hazards: the road transport environment for conveyance of hazardous materials in Great Britain”, J. of Haz. Mat., 32, pp. 41-79, 1992

18. I.A. Papazoglou, G.S. Bonanos, Z.S. Nivolianitou, N.J. Duijm, B. Rasmussen, "Supporting decision makers in land use planning around chemical sites. Case study: expansion of an oil refinery", J. of Hazardous Materials, 71, pp 343-373, 2000

19. K.H. Harrington, S.E. Rose, "Wisely make investment decisions via risk mapping”, Chemical Engineering Progress, 95/12, 63-66, 1999

20. Fabiano, F. Currò, E. Palazzi , R. Pastorino, "A framework for risk assessment and decision-making strategies in dangerous good transportation”, J. of Loss Prevention in the Proc Ind., 93, pp. 1-15, 2002

21. F.I. Khan, S.A. Abbasi, "Inherently safer design based on rapid risk analysis", J. of Loss Prevention in the Proc Ind., 11, pp. 361-372, 1998

22. CCPS, Center for Chemical Process Safety, "Guidelines for Chemical Transportation Risk Analysis", New York: AIChE, 1995.

23. R. Bubbico, S. Di Cave, and B. Mazzarotta, "Risk analysis for road and rail transport of hazardous materials: a simplified approach”, J. of Loss Prevention in the Proc Ind., Vol 17/6, pp. 477-482, 2004.

24. C.B. Goh, C.B. Ching, R. Tan, "Risk analysis for the road transportation of hazardous chemicals in Singapore - a methodology”, J. of Loss Prevention in the Proc Ind., 8, pp. 35-39, 1995

25. Fabiano, F. Currò, A.P. Reverberi, R. Pastorino, "Dangerous good transportation by road: from risk analysis to emergency planning”, J. of Loss Prevention in the Proc Ind., 18, pp. 403-413, 2005

26. R. Bubbico, S. Di Cave, B. Mazzarotta, "Risk analysis for road and rail transport of hazardous materials: a GIS approach", J. of Loss Prevention in the Proc Ind., Vol 17/6, pp. 483-488, 2004.

27. R. Bubbico, S. Di Cave, B. Mazzarotta, "La gestione del rischio nel trasporto di merci pericolose via terra”, Convegno Scientifico Nazionale "Sicurezza nei sistemi complessi”, III edizione, Bari, 19-21 ottobre 2005, Sessione III/2, (in Italian), 2005.

28. R. Bubbico, G. Maschio, B. Mazzarotta, M.F. Milazzo, E. Parisi, "Risk management of road and rail transport of hazardous materials in Sicily", J. of Loss Prevention in the Proc Ind., Vol 19/1, pp. 32-38, 2006.

29. G. Purdy, "Risk analysis of the transportation of dangerous goods by road and rail”, J of Haz. Mat., 33, pp. 229-259, 1993 